British Journal of Psychiatry (1993), 162, 564-575

\title{
Correspondence
}

Editor: Ian Pullen

Contents: Puerperal affective psychosis: is there a case for lithium prophylaxis?/Assessment of insight in Singapore/Clozapine and NMS/In defence of clozapine/Clozapine, cognition, and schizophrenia/ Mentally ill sex offenders/Mabi bark tea/Genetic basis for transsexualism/'Hidden' spending on community services/Professional scepticism towards multiple personality disorder/HIV infection, serotonin, and sexual dysfunction/Hysteria in childhood/Hysterical conversion and developmental psychiatry/Folate and depression/Outdated ECT machines/ECT in schizophrenia: need for reappraisal?/Parental age in schizophrenia in a case-controlled study.

\section{Puerperal affective psychosis: is there a case for lithium prophylaxis?}

SIR: I read with interest Austin's research report (Journal, November 1992, 161, 692-694) where she concluded that lithium prophylaxis in puerperal affective psychoses prevented significant post-partum relapse in contrast to no medication. Stewart (1988) advocated lithium treatment immediately after delivery for women with bipolar illness or a history of puerperal psychoses. This approach probably prevented some relapses.

We also have experience of using medication (lithium, haloperidol, or carbamazepine) immediately after delivery for bipolar patients. In our study, $27 \%$ of patients ( 3 out of 11) who used prophylaxis after delivery had a relapse (manic or depressive) in the first three months, compared with $60 \%$ of the patients (3 out of 5) who refused medication (van Gent \& Verhoeven, 1992). One patient who became manic, in spite of an adequate serum lithium level $(0.82 \mathrm{mmol} / \mathrm{l})$, had used bromocriptine to reduce lactation. Indeed, some authors (Kemperman \& Zwanikken, 1987) have reported mania associated with bromocriptine. Since then, in our protocol for lithium prophylaxis after pregnancy, we have warned against inhibiting lactation by means of bromocriptine.

Kemperman, C. J. F. \& Zwantkken, G. J. (1987) Psychiatric sideeffects of bromocriptine therapy for postpartum galactorrhoea. Journal of the Royal Society of Medicine, 80, 387-388.
Stewart, D. E. (1988) Prophylactic lithium in postpartum affective psychosis. Journal of Nervous and Mental Disease, 176, 485-489. van Gent, E. M. \& Verhoeven, W. M. A. (1992) Bipolar illness, lithium prophylaxis and pregnancy. Pharmacopsychiatry, 25, 187-191.

EduARd M. van Gent

Lithium Out-patient Clinic

Willem Arntsz Huis

P.O. Box 85398, 3508 AJ Utrecht

The Netherlands

SIR: The interesting retrospective study by Austin (Journal, November 1992, 161, 692-694) suggested that lithium is effective in reducing the risk of recurrence of puerperal psychosis after subsequent pregnancies. One of us (McKenzie, 1991) has recently completed an outcome study of women suffering from post-partum psychosis who were admitted to a mother-and-baby unit in Manchester between 1978 and 1988. Only women who were suffering from a first episode of psychosis were included. A small number of the study group were discharged on lithium ( 9 out of 69 ). However, in the six months following discharge, none of the patients on lithium had a relapse, whereas almost half the patients not receiving lithium relapsed (27 out of $60 ; P=0.02$, $\chi^{2}$ test), many needing readmission (16 out of 60 ; $P=0.09, \chi^{2}$ test). The difference occurred despite more severe illness in those discharged on lithium, as reflected in their longer admission. These findings suggest lithium is indicated in the treatment of first episodes of puerperal psychosis, in addition to its prophylactic use, as suggested by Austin.

Almost half of the women (34 out of 69) had a subsequent episode of illness within six years. This is likely to be an underestimate since some cases were followed up for a shorter period of time, and episodes of illness in women who moved out of the area were not recorded. The high rate of recurrence suggests the need for prophylaxis. However, in this study most of the further episodes of illness were unrelated to the puerperium (43 non-puerperal episodes compared with 13 post-partum). Previous studies have had similar findings (Protheroe, 1969; Lindsay \& 